\title{
Concentric Joint Connectors for Form-Changing Space Frames
}

\author{
P. Harkin \\ Form Changing Structures Limited and Imperial College London \\ R. Vaidyanathan \\ Imperial College London \\ S. Morad \\ Aston University, UK
}

\begin{abstract}
This paper presents a novel joint design for use in form-changing structural frames. These structures are comprised of multiple struts, arranged in a geometrically stable array, connected at their end points via the joints. Each strut, acting as part of a truss, can change in length (telescope), and thus their combined variations can change the overall form of the frame. The joints enable multiple struts to be connected together and pivot around each node point. The joints have near-perfect concentricity of rotation around the node point, which enables the tension and compression forces of the struts to be resolved in a structurallyefficient manner. The design evolved from an analysis of simple, pure-tension net structures, combined with aspects of rigid space frame structures, to resist compressive forces. Existing joint configurations are analyzed and compared to the novel joint. Several applications of form-changing structural frames are noted, to demonstrate the advantages of using such joint connectors in these frames; one is a variable-geometry mount for a surgical device, for Imperial College London.
\end{abstract}

\section{INTRODUCTION}

\subsection{Space frames}

Space frame structures emerged in the 1890s, from work by Bell (1903) related to structures for kites that were lightweight, but also strong. They are comprised from rigid struts joined together at their ends, configured as 3-dimensional trusses. Figure 1 shows examples based on struts in square pyramidal/tetrahedral and octahedral/tetrahedral arrangements. Historically, these frames have used struts of fixed lengths to create rigid structures.
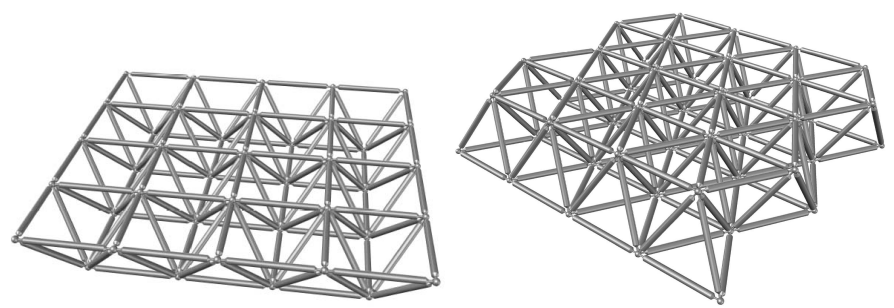

Figure 1. Space frames - pyramidal/tetrahedral (left) \& tetrahedral/octahedral (right) arrangements

\subsection{Space frame joints}

Figure 2 shows a close-up view of a rigid space frame, comprised of fixed-length struts (S). The angular displacement (AS) between all the struts does not change; therefore the struts can be connected at the vertices (or nodes) via simple rigid joints at the convergence points $(\mathrm{CP})$, which are often spherical in form $\left(\mathrm{J}_{\mathrm{S}}\right)$.

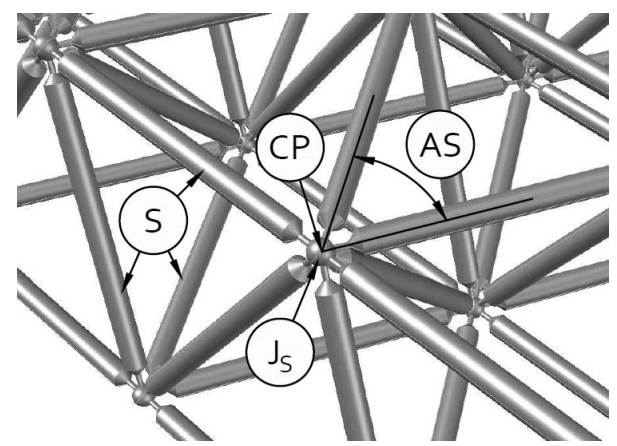

Figure 2. Space frame node with rigid joint

Figure 3 shows a simplified, detailed view of the same fixed spherical joint (JS). It is important that the struts' axes, and therefore the lines of force (LF) all need to intersect at the convergence point (CP) at the nodes. From Lan (1999), this alignment, along with the use of triangulation in 3 dimensions, means loads imposed upon the structure are resisted almost 
wholly via tension forces $\left(\mathrm{F}_{\mathrm{T}}\right)$ or compression forces $\left(\mathrm{F}_{\mathrm{C}}\right)$ along the axes of the struts forming the frame, with little or no shear or bending forces acting upon the struts. Thus the struts only have to resist elongation from tension, or buckling due to compression. This leads to a very efficient structure for a given quantity of material, i.e. they have excellent strength-to-weight ratios.

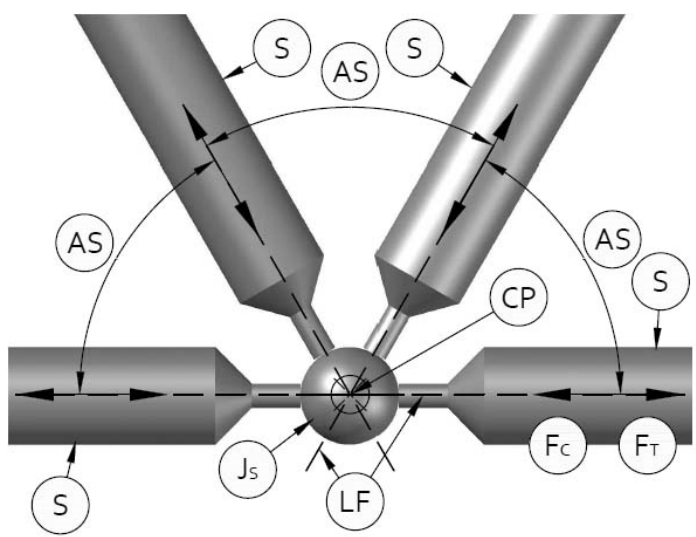

Figure 3. Space frame node - rigid joint detail

It will be seen that there are at least 3 struts converging at any of the nodes within a space frame, and this can increase to 8 in a pyramidal/tetrahedral arrangement of the frame, or 9 struts in the tetrahedral/octahedral frame, both shown in Figure 1. If the frame is double-layered, up to 12 struts converge at the nodes for either arrangement.

\section{FORM-CHANGING SPACE FRAMES}

\subsection{Overview}

A distinctive type of form-changing space frame structure has emerged in engineering design since the 1940s; these can act as both a rigid structure in a variety of fixed forms, or continuously vary their form, depending on the requirements of a wide variety of applications. These have struts that can change their length (or 'telescope') in a controlled manner (i.e. they are linear actuators), connected together pivotally at their ends, and arranged in the same geometrically-stable forms as rigid space frames. By varying the length of the struts, the frame can assume a wide variety of forms.

A widely-used version of this type of structure is the Gough/Stewart Platform, originally developed by Gough (1962) as a tyre-testing device in the 1950 s \& 60s. These typically comprise upper and lower rigid plates, connected via 6 telescopic struts, and are referred to with terms such as 'parallel hexapod manipulators' in robotics. However, since the $1990 \mathrm{~s}$, there has been growing interest in frames comprised entirely of telescopic struts, with no rigid sections, and often with multiple 'cells' of struts forming arrays of tetrahedrons and/or octahedrons, which are inherently able to assume a wide variety of forms.

\subsection{Potential applications}

Although largely theoretical at present, these form-changing structures, optionally clad with a flexible skin (Figure 4) have many potential applications, such as adaptive airline seating, morphing car exteriors and interiors, emergency structural support structures, variable-shape moulds/jigs for forming other materials etc. Figure 5 shows a working prototype of a form-changing octahedral structure developed by the author.
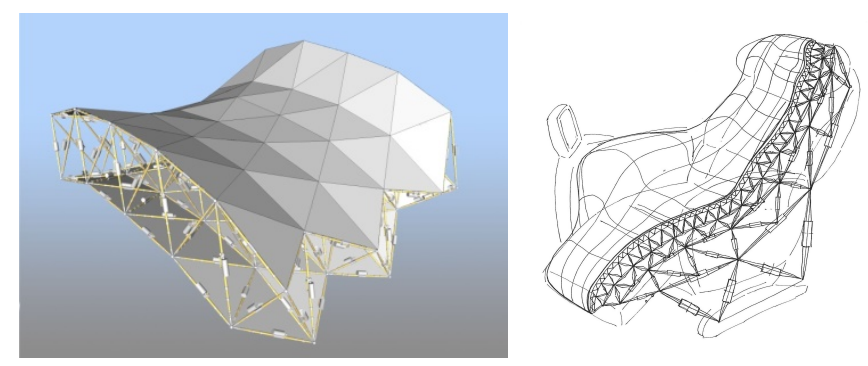

Figure 4. Form-changing space frame structures with optional skin

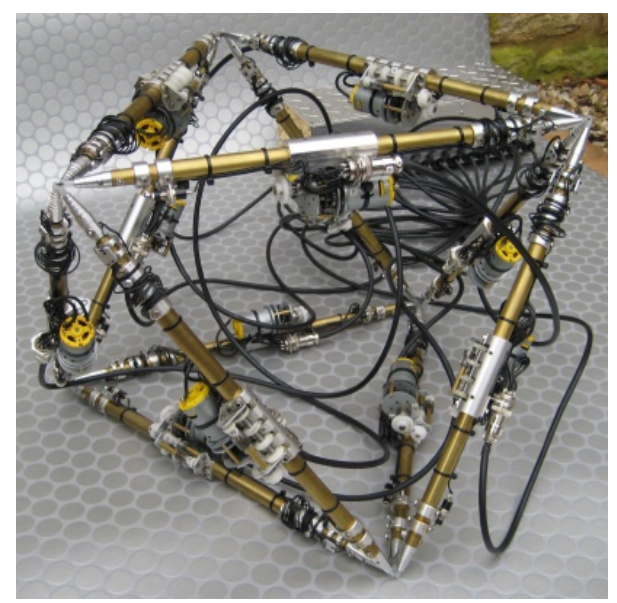

Figure 5. Prototype octahedral form-changing space frame

\subsection{Requirements for the joints}

In a form-changing space frame, the struts can change length independently of one another. Therefore, at each node point, a joint is required where at least 3 struts need to pivot with 3 degrees of freedom (DOF) around the nodes where the ends of the struts are connected. The pivot point needs to be at the node point of the space frame to ensure the struts form the triangles that are key to the frames stability and structural efficiency (i.e. the pivot points must be concentric). In both nature and man-made objects, there are countless joint mechanisms that connect together 2 elements that can pivot around a point with 3 DOF, e.g. the ball-and-socket joint found in the human shoulder. Joints that enable this for 3 or more convergent struts are not thought to 
exist in nature, at least not with a close degree of concentricity of the pivot points, so inspiration cannot be sought from there. Neither do they appear to have been developed beyond the prototype stage in the field of mechanical engineering.

\section{JOINT SOLUTIONS}

\subsection{Initial ideas}

When first considering the design of the joints for a form-changing space frame, several solutions appeared to be suitable. Initially, crude prototypes were made using flexible rubber tubing, with the ends of the tubes cut with a scalpel to match the form of the adjoining section, with the sections glued to each other, and then assembled with the struts as part of a frame (Figure 6). Whilst this was acceptable for the purposes of an initial proof-ofconcept prototype, failure of the joints was common, often due to the de-bonding of the glued sections.

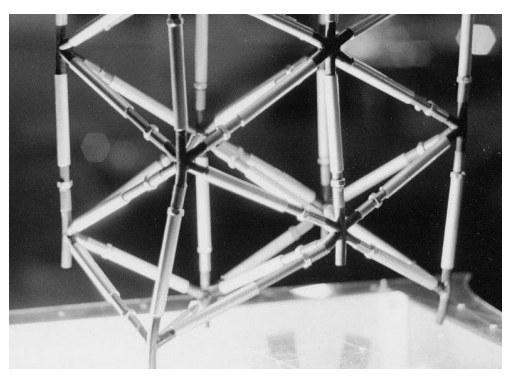

Figure 6. Initial prototype frame and joints

Single piece joints were moulded in silicone rubber to avoid the need to bond the sections together, which were less prone to failure, but their strength was limited by the moulded material. For both examples, the pivoting of the struts was imprecise and not concentric around the frame's node points.

\subsection{Ball and socket joints - inherent disadvantages}

Figure 7 shows a joint assembly of 3 DOF balland-socket joints $\left(\mathrm{J}_{\mathrm{BS}}\right)$. The non-concentric pivot points (PP) of the joints are separated by distance D. The separation is necessary as this mechanism is not able to be transposed into the same volume as another. The forces of compression $\left(\mathrm{F}_{\mathrm{C}}\right)$ and tension $\left(\mathrm{F}_{\mathrm{T}}\right)$ acting along the axes of the struts are shown as lines of force (LF). With the angles of separation (AS) between the struts as shown, the lines of force intersect at a convergence point $(\mathrm{CP})$ of the central connection (CC), and the struts are not subject to shear or moment forces.

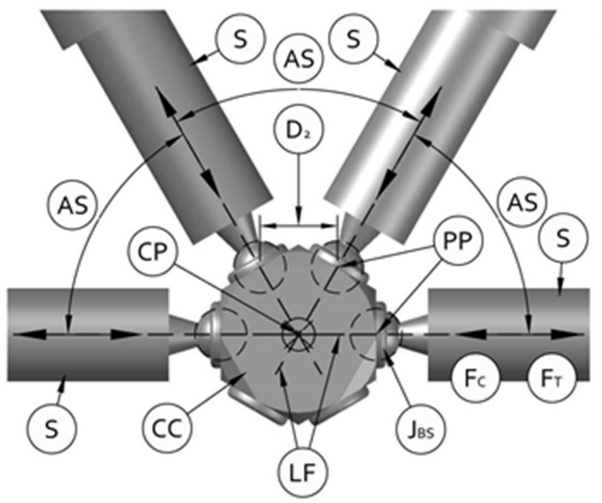

Figure 7. Ball and socket joints - aligned

Figure 8 shows the same joint arrangement, but with forces of compression $\left(\mathrm{F}_{\mathrm{C}}\right)$ acting upon the struts (S). The central connection (CC) component has been made to rotate due to the loads from the struts acting as moment forces $\left(\mathrm{F}_{\mathrm{M}}\right)$ upon it. The lines of force (LF) now do not pass through the convergence point $(\mathrm{CP})$. At the ball-and-socket joint $\mathrm{J}_{\mathrm{BS} 1}$ the strut $\left(\mathrm{S}_{1}\right)$ connected to the ball is now in contact with the socket edge of the socket at point $\mathrm{P}_{1}$. Thus a load resulting in a compression force $\left(\mathrm{F}_{\mathrm{C}}\right)$ upon strut $\mathrm{S}_{1}$ would impose a bending moment at this point of contact $\left(\mathrm{P}_{1}\right)$ upon the end of strut $\mathrm{S}_{1}$ as it induces further rotation of the central connection. The intrinsic structural efficiency of the space frame structure is compromised, as its struts now have to resist shear and bending forces, rather than simply resisting tension $\left(\mathrm{F}_{\mathrm{T}}\right)$ and compression $\left(\mathrm{F}_{\mathrm{C}}\right)$ loads along their primary axes.

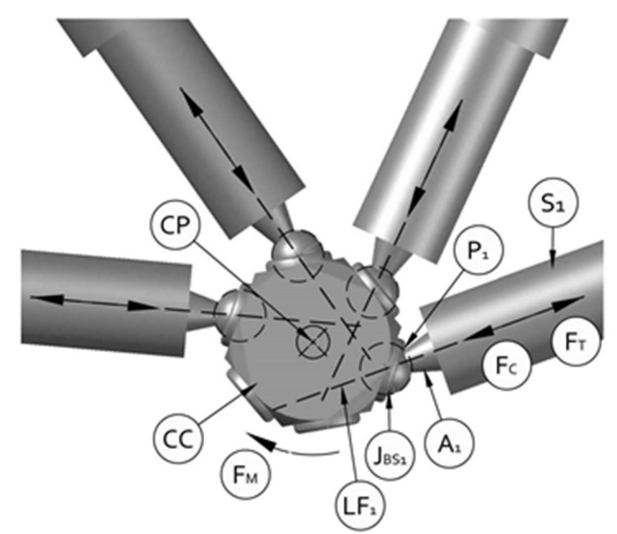

Figure 8. Ball and socket joints - misaligned

Should the loads on the struts (S) change to result in tension forces $\left(\mathrm{F}_{\mathrm{T}}\right)$, the central connection will rotate back in the opposite direction, as the struts exert a pulling force upon it via the ball/socket joints. This rotation could be sudden, and difficult for any control system to predict. This rotation amounts to localised instability of the joint assembly. For the overall framework, the accumulation of these localised instabilities would lead to wider patterns of more pronounced and uncontrolled instability. 


\subsection{Mechanisms for concentric joints}

As part of the growing interest in variable geometry truss structures within the robotic community since the 1990s, several research groups have been developing novel designs for truly concentric freerotation joints (Fig. 9), such as those by Bosscher/Ebert-Uphoff (2003) and Hamlin (1994).
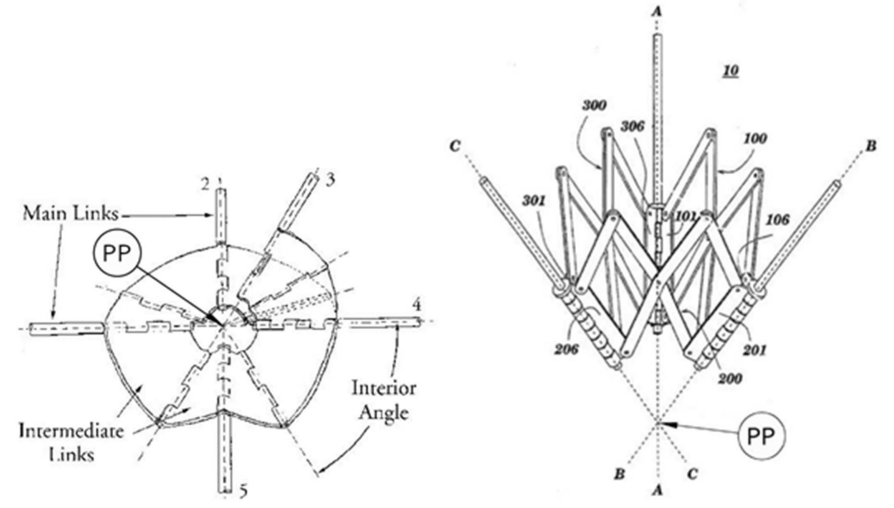

Figure 9. Concentric joints by Bosscher/Ebert-Uphoff (left) and Hamlin (right)

When attempting to design such a concentric 3 DoF joint for 3 or more convergent struts, it turns out to be a deceptively difficult task. Instinctively, the designer may wish to locate rigid material precisely at the pivot point (i.e. the vertices/nodes), in order to transfer loads efficiently and within a compact form. Yet the relative movement of more than two connected struts around a shared pivot point (i.e. concentricity) results in the connecting material or mechanism of each strut attempting to overlap in space with the other connections, and the capability for movement can be lost. Both of the joints shown above project a virtual pivot point (PP) into space outside of its own structure, making this a shared pivot point for all of the convergent struts.

However, both of these examples use relatively bulky and complex mechanisms, with many moving parts. Instead, a concentric joint design was sought that was simple, compact, robust, and used few, if any, moving parts.

\section{PATH TOWARDS A SOLUTION}

\subsection{Tension structures}

For the resolution of purely tension loads, multi-link concentric joints that can resolve tension forces, but not compression, have been in common use for millennia - one example being those within a structure woven from flexible fibres or cords, such as a fishing net.

However, for the many potential applications where a form-changing space frame structure might be used, both tension and compression forces occur within the struts. Hence both types of loads must be resolved by the nodal connections.

\subsection{Resolving tension and compression forces}

A way was sought to utilise the simplicity and efficiency of a tension-based network comprised entirely of flexible cords, yet also have it resist compressive forces. The key insight was to re-examine the joints within rigid space frame structures, and understand how these resisted compressive forces, and then see if the principles could be combined with the efficiency and simplicity of the tension cords.

For the example shown in Figure 2, the frame's struts join together at their ends via their connection to solid blocks, which are typically spherical in form. For commercially-available rigid space frames, the spheres act as a convenient method of joining the struts together, thus extending the material structure of each strut towards the node point, where the tension/compression forces are transmitted to the conjoining struts. Instead of the sphere, the struts themselves could be extended as close as practical to the node point, and joined directly to the other adjoining struts, such as by welding or bonding (i.e. gluing). As the struts are subject to compressive forces along their lengths, the ends at the joints have a tendency to push onto the adjacent strut end. In effect, the strut is attempting to push through and then past the ends of the other struts.

\subsection{Use of cords to tie struts together}

But what if the joining by bonding of the strut ends could be done via the flexible cords of the net structure, passing through the ends of the struts, and entwining with other cords from other struts, thus tying the ends together? A compressive load, pushing along the axis of the strut, would, as above, be induced to push the end of the strut through and then past the ends of the other struts, but the cord would prevent it from doing so. When the strut is subject to a tension force, the cord would resist this by itself going into tension. Due to the ends of the struts not being joined rigidly to each other, but only pulled into contact with each other by the flexible cord, they can effectively pivot relative to each other, giving 3 DOF. Figure 10 shows a cross-section view through the joint configuration. This shows the ends of the struts (S) as tubular forms, with collars (CL) inserted in their ends, which in turn have lengths of cords (C) passing through them. The cords are knotted at their ends $(\mathrm{KN})$ to prevent them passing through the collars, and are entwined with other cords to retain the strut ends. 


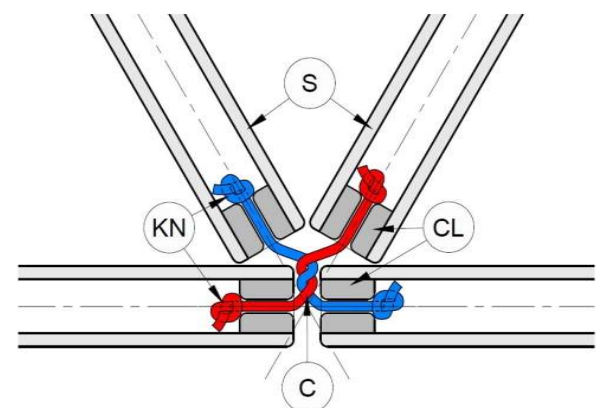

Figure 10. Cross-section of struts tied together with cords

\subsection{Refinements to the design}

This crude arrangement would function as a $3 \mathrm{DOF}$ joint for 3 or more struts, but there are some important refinements that can be made to greatly improve the joint's performance, shown in Figure 11.

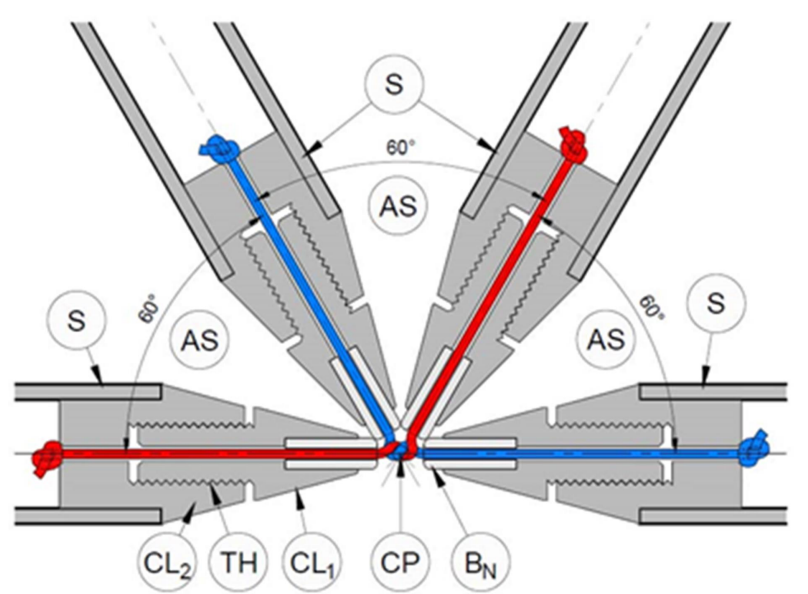

Figure 11. Cross-section of tapering cord joint

As the tips of the struts are in moving contact with one another, the extremities of struts have been formed as relatively short tubular barrels of a lowfriction bearing material $\left(\mathrm{B}_{\mathrm{N}}\right)$. Nylon 66 (a type of polyamide) was chosen in prototypes due to its balance between reasonable resistance to bending forces, low friction properties and good resistance to impact loading; it was accepted that relatively minor deformations of the nylon tips would add to the joints overall robustness, with only a negligible effect on the joints overall concentricity within the frame. The cord passes through the barrel, emerging to entwine with other cords. A relatively non-brittle material such as nylon is also suitable as it directly contacts the cord and exerts a pressure upon it, e.g. when the strut is subject to a compressive force, it attempts to push past the other barrels and shear the cord.

A 2-piece end collar $\left(\mathrm{CL}_{1}, \mathrm{CL}_{2}\right)$ with a conical form is located at the end of each strut (S); i.e. their cross-sectional area decreases towards the node point. This provides rigid support for the barrel and in turn the cord very near to the convergence point at the node. This partially overcomes the issue noted previously; i.e. the seemingly conflicting desire to locate rigid materials as close as practically possible to the point where the struts join together, whilst still allowing clearance for the struts to pivot around the node point.

\subsection{Cord tension adjustment and elasticity}

Collars $\mathrm{CL}_{1}$ and $\mathrm{CL}_{2}$ are threaded together; their rotation relative to each other varies the distance between the cord knot $(\mathrm{KN})$ and the barrel $\left(\mathrm{B}_{\mathrm{N}}\right)$, thus giving a convenient method of adjusting the tension in the cords.

It is important factor for the cords to have a slight degree of elasticity whilst the struts pivot around the node point, its length would need to vary slightly depending on the angular displacement of the struts. The degree of this elasticity would have to be chosen carefully, as it would need to balance sufficient 'slackness' to allow the struts to pivot freely versus ensuring the strut ends maintain contact with one another. The elasticity would also have to be chosen to resist the forces acting on the joint i.e. the tensile/compressive forces imposed by the struts.

\subsection{Failure of the joint}

It is possible that the joint would ultimately fail due to compression loading once the cord elongated to such a degree that the tip of one strut passed beyond that of the others, leading to two eventualities. One is that the cord would be elongated beyond its elastic limit and its elongation becomes irreversible (i.e. plastic), then when the compressive load is removed, the strut end would no longer be in contact with the adjacent strut ends, leading to loss of concentricity. The second is that the 'barrel' tubular form at the end of the strut would be subject to a concentrated bending moment as the cord would still be restrained by the other struts, likely leading to its permanent deformation and/or disintegration.

Failure due to tension loading would occur when the cord elongates beyond its elastic limit; when the load was removed, this would lead to a similar lack of contact between the strut ends as above.

Obviously, in addition to the cord elongating beyond its elastic limit in either scenario noted above, the ultimate failure of the joint would occur should the cord break. In the prototype joints, the cord chosen ('Beta Saltwater Mono 80lb Clear' by Shakespeare Fishing) was a monofilament fishing line of $1.2 \mathrm{~mm}$ diameter, with a breaking load of $36 \mathrm{~kg}$, which also has a sufficient degree of elasticity to allow the struts to pivot. Testing of the line showed it elongated approx. $1.5 \mathrm{~mm}$ when supporting a $5 \mathrm{~kg}$ load. 


\subsection{Concentricity}

It is acknowledged that the proposed joint design has less than perfect concentricity of the pivot points of the struts. Figure 12 shows that the actual pivot point (PP) is not at the original convergence point (CP), but closer to the rounded contact point between the barrels $\left(\mathrm{B}_{\mathrm{N}}\right)$ at the ends of the struts. In the built prototypes, the distance between the node point and the actual pivot point was approximately $4 \mathrm{~mm}$. This displacement of concentricity appears to be proportional and approximately equal to the diameter of the barrels $(4 \mathrm{~mm})$. This displacement distance is smaller than could practically be achieved using multiple ball-and-socket joints. It is also proportionally low relative to the overall length of the telescopic struts of the prototypes (which vary in length from $450 \mathrm{~mm}$ to $270 \mathrm{~mm}$ ); the displacement of concentricity is between $0.9 \%$ and $1.5 \%$ of the strut length.

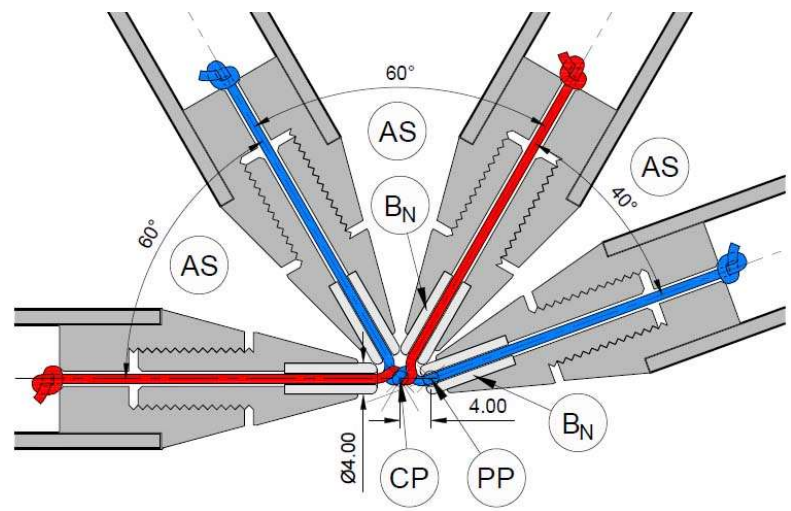

Figure 12. Cross-section of tapering cord joint, with strut pivoting

\section{CONCLUSIONS}

\subsection{Overview}

Tapering Cord Joints appear to be well suited for use in variable-geometry truss structures, for both existing applications such as hexapods/Stewart Platforms in surgical/industrial devices, and more speculative applications such as form-changing space frames (which are mostly in the working-prototype stage). This is due to their combination of nearconcentricity, relatively few moving parts, and their rugged and simple overall design.

\subsection{Operation in various environments}

Consideration would need to be given to the environment in which the joints would eventually operate. Sealing against the ingress of dirt, dust or grit is likely to be necessary in some circumstances, e.g. a flexible covering or encasement. It is not anticipated that water ingress would significantly affect the joint's performance, provided material such as nylon was used for bearing components.

\subsection{Possible design improvements}

Due to the proportional relationship between the diameter of the barrels at the strut ends and the offset of the strut pivot points from the node points, there appears to be scope for the pivot points to be closer to the nodes, thus moving closer to trueconcentricity, via the use of materials for the barrels with higher flexural strength. This would allow them to be smaller in overall diameter and cross-sectional area for a given yield value; the joint components could be more tightly compacted together, whilst still enabling sufficient freedom of movement.

\subsection{Early applications and future plans}

The joints as manufactured (Figure 13) performed very well as part of the octahedral form-changing space frame (Figure 5) and a prototype hexapod manipulator surgical platform developed with Morad (2014) at Imperial College London. It is hoped that they be developed further as key components of multi-strut form-changing space frame structures in the future. The Tapering Cord Joint design is the subject of a WIPO patent application (ref. WO2014006422).
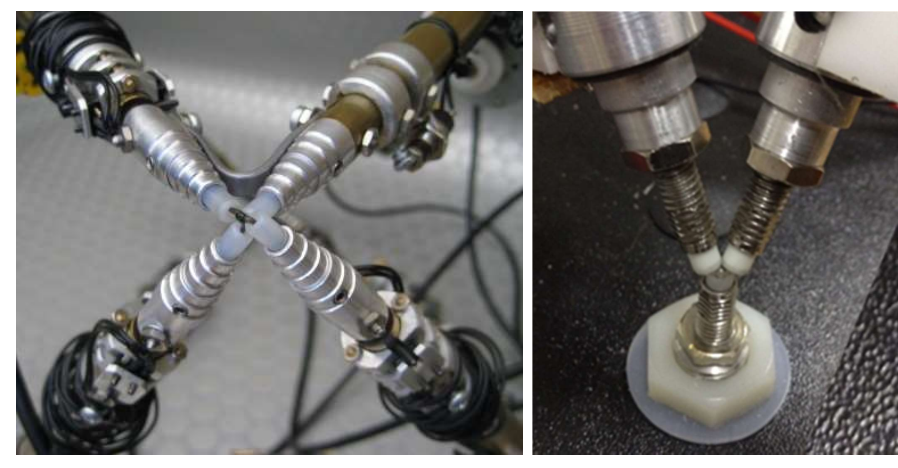

Figure 13. Tapering Cord Joints as part of a prototype octahedral-frame (left) and a prototype surgical platform (right)

\section{REFERENCES}

Bell, A.G. 1903. Tetrahedral Principle in Kite Structure. In National Geographic Magazine Vol. XIV, No.6. Washington, D.C. USA: National Geographic Magazine

Bosscher, P. \& Ebert-Uphoff, I. 2003. A Novel Mechanism for Implementing Multiple Collocated Spherical Joints Georgia, USA: Georgia Institute of Technology

Gough, V.E. \& Whitehall, S.G. 1962. Universal tyre test machine. In: Proceedings of the FISITA 9th international technical congress, London: 117-137. London: Institution of Mechanical Engineers.

Hamlin, G. et al 1994. A Novel Concentric Multilink Spherical Joint with Parallel Robotics Applications Troy, NY, USA: Rensselaer Polytechnic Institute.

Lan, T.T. 1999. Space Frame Structures. In Chen Wai-Fah (ed), Structural Engineering Handbook: 13.1.4. Boca Raton: CRC Press LLC

Morad, S. et al 2014. Flexible robotic device for spinal surgery In: IEEE International Conference on Robotics and Biomimetics Bali, Indonesia: IEEE. 